\section{Adaptive Regulation of Amplitude Limited Robot Manipulators With Uncertain Kinematics and Dynamics}

\author{
W. E. Dixon
}

\begin{abstract}
Common assumptions in most of the previous robot controllers are that the robot kinematics and manipulator Jacobian are perfectly known and that the robot actuators are able to generate the necessary level of torque inputs. In this note, an amplitude-limited torque input controller is developed for revolute robot manipulators with uncertainty in the kinematic and dynamic models. The adaptive controller yields semiglobal asymptotic regulation of the task-space setpoint error. The advantages of the proposed controller include the ability to actively compensate for unknown parametric effects in the dynamic and kinematic model and the ability to ensure actuator constraints are not breached by calculating the maximum required torque $a$ priori.
\end{abstract}

\section{INTRODUCTION}

For a robotic system to interact with and execute tasks in the taskspace, a transformation between the position and orientation of the robot with respect to objects located in the task-space is typically required. Since the robot is controlled through inputs to the link actuators, the robot kinematics and manipulator Jacobian are used to relate a task-space coordinate system to coordinate systems attached to each actuator (i.e., the joint-space). A common assumption in most of the previous robot control literature is that the kinematics and manipulator Jacobian are perfectly known. This assumption limits robustness because uncertainty in the robot kinematics and Jacobian may lead to degraded performance or unpredictable responses.

Uncertainty in the kinematic model of a robot is a practical problem. The end-effector of a manipulator is often interchanged with other end effector tools with different lengths; the manipulator picks up an object with an unknown length with the intention of using it as a tool; or generic robot data sheets may be used that are not specific to the particular robot. For example, the Puma 560 has well published kinematics but many different versions of the robot are on the market that have the same kinematic structure but different kinematic parameters (e.g., due to differences in the manufacturers). From these examples, it is also clear that uncertainty in the kinematic model is a separate problem from uncertainty in the dynamic model. In the invited paper [1], Arimoto describes the importance of the problem with uncertain kinematic parameters and states that research which targets this problem is at the beginning stages.

The observations by Arimoto are supported by a review of literature which yields relatively few different approaches to address this topic. In [28], Shahamiri and Jagersand used a nullspace-biased Newton-step visual servo strategy with a Broyden type Jacobian estimation for on-line singularity detection and avoidance in an uncalibrated visual servo control problem. Based on a similar idea, Piepmeier et al. published a se-

Manuscript received August 5, 2005; revised April 12, 2006 and August 31, 2006. Recommended by Associate Editor A. Astolfi. This work was supported in part by the National Science Foundation under CAREER Award CMS-0547448, by the Air Force Office of Scientific Research under Contracts F49620-03-1-0381 and F49620-03-1-0170, by the AFRL under Contract FA4819-05-D-0011, and by Research Grant US-3715-05 from BARD, the United States-Israel Binational Agricultural Research and Development Fund at the University of Florida.

The author is with the Mechanical and Aerospace Engineering Department, the University of Florida, Gainesville, FL 32611-6250 USA (e-mail: wdixon@ufl.edu).

Color versions of one or more of the figures in this paper are available online at http://ieeexplore.ieee.org.

Digital Object Identifier 10.1109/TAC.2006.890321 ries of papers (e.g., see [21]-[25]) that exploit dynamic quasi-Newton recursive least-squares estimators to solve a variety of visual servo control problems despite an uncertain kinematic model and uncertainty in the camera model.

Some results have been published by Cheah et al. in [4]-[6] that address uncertainty in the kinematic model. Specifically, Cheah et al. developed several approximate Jacobian feedback controllers in [4]-[6] that exploit a static, best-guess estimate of the manipulator Jacobian to achieve task-space regulation objectives despite parametric uncertainty in the manipulator Jacobian. As reported in [3], a drawback of the controllers in [4]-[6] is that the task-space velocity of the robot end-effector is required to be measurable, and the controller in [5] requires the computation of an estimate for the Jacobian inverse. In [3], Cheah et al. resolve these issues by developing a controller that exploits a static, best-guess estimate of the manipulator Jacobian to achieve a task-space regulation result.

Controllers that exploit combinations of proportional, integral, or derivative feedback terms (i.e., PD and PID) such as the seminal work in [19] can be used to achieve joint-space regulation in the presence of uncertainty in the dynamic model; however, task-space regulation has yet to be proven by such a controller when the kinematics and manipulator Jacobian are corrupted by uncertainty. A PID controller such as [19] could be used to achieve joint-space regulation that is coupled with an adaptive path planner that compensates for the kinematic uncertainty. This is a valid approach that is also at the beginning stages of research with some results presented in [7] and [14]; however, shifting the ability to compensate for the kinematic uncertainty on the path planner may not be reasonable for some applications. That is, efforts should also be developed that compensate for the uncertainty through the controller so that a generic path planner can be easily incorporated.

The controller developed in this note (and the preliminary work in [12]) is the first adaptive result for the regulation problem under the presence of kinematic and dynamic uncertainty. As in the recent result in [3], the controller does not require the task-space velocity of the robot end-effector to be measurable, does not require the inverse of the estimated Jacobian to be computed, and does not require exact model knowledge of the robot dynamics. The current result actively compensates for uncertainty in the gravity and static friction effects. In contrast to the use of a static, best-guess estimate as in [3], the controller in this note also exploits a feedforward control term that actively compensates for the parametric uncertainty in the Jacobian. The adaptive controller in this note provides the first result that eliminates the assumption (required by the previous robust controllers) that the mismatch between the Jacobian and the Jacobian estimate be bounded as follows:

$$
\|J(q)-\hat{J}(q)\| \leq \rho
$$

where $\rho$ is some known positive constant. Moreover, the constraints on the feedback gains that are imposed by (1) (i.e., high-gain robust feedback) are eliminated by the proposed adaptive controller. The development of the adaptive controller requires an innovative arrangement of the uncertainty in the open-loop error system that is enabled by using appropriate saturation methods to bound functions in a specific manner. Strategic use of the saturation functions coupled with an innovative nonquadratic Lyapunov function also allows the control effort to be a priori bounded.

The fact that the controller can be a priori bounded is a significant added advantage. The practical implications are that the actuators can be appropriately sized (or the control gains set to specific thresholds) without requiring an ad hoc saturation scheme (that would not be included in the stability analysis) to protect the actuator. The common assumption that the robot actuators are able to generate the necessary 
level of torque is limiting since robotic actuators have physical constraints. If the controller commands more torque than the actuators can supply from typical control methods, degraded or unpredictable motion control and thermal or mechanical failure may result. For a review of literature that addresses amplitude limited control designs, see [8]-[10], [13], [16], [17], [26], [30], and the references within.

\section{ROBOT MODEL}

The dynamic model for a rigid $n$-link, serially connected, directdrive revolute robot is given as follows [15]:

$$
M(q) \ddot{q}+V_{m}(q, \dot{q}) \dot{q}+G(q)+F_{s} \operatorname{sgn}(\dot{q})=\tau .
$$

In (2), $q(t), \dot{q}(t), \ddot{q}(t) \in \mathbb{R}^{n}$ denote the link position, velocity, and acceleration vectors, respectively, $M(q) \in \mathbb{R}^{n \times n}$ represents the inertia matrix, $V_{m}(q, \dot{q}) \in \mathbb{R}^{n \times n}$ represents centripetal-Coriolis matrix, $G(q) \in \mathbb{R}^{n}$ represents gravity effects, $F_{s} \in \mathbb{R}^{n \times n}$ denotes the constant diagonal static friction matrix, $\operatorname{sgn}(\cdot) \in \mathbb{R}^{n}$ denotes the vector signum function, and $\tau(t) \in \mathbb{R}^{n}$ represents the torque input vector. Let $x(t) \in \mathbb{R}^{m}(m \leq n)$ represent a task-space vector that is related to the robot joint-space as

$$
x=h(q) \quad \dot{x}=J(q) \dot{q}(t)
$$

where $h(q) \in \mathbb{R}^{m}$ denotes the differentiable forward kinematics of the manipulator, and $J(q) \triangleq(\partial h / \partial q) \in \mathbb{R}^{m \times n}$ denotes the differentiable manipulator Jacobian.

The dynamic model introduced in (2) has the following properties that are used in the subsequent control development and analysis.

Property 1: The positive-definite and symmetric inertia matrix, satisfies the following inequalities [11]:

$$
m_{1}\|\xi\|^{2} \leq \xi^{T} M(q) \xi \leq m_{2}\|\xi\|^{2} \quad \forall \xi \in \mathbb{R}^{n}
$$

where $m_{1}, m_{2} \in \mathbb{R}$ are known positive bounding constants, and $\|\cdot\|$ is the standard Euclidean norm.

Property 2: The time derivative of the inertia matrix and the centripetal-Coriolis matrix satisfy the following skew symmetric relationship [11]:

$$
\xi^{T}\left(\frac{1}{2} \dot{M}(q)-V_{m}(q, \dot{q})\right) \xi=0 \quad \forall \xi \in \mathbb{R}^{n} .
$$

Property 3: The unknown gravitational and static friction terms can be linearly parameterized as follows [11]:

$$
Y(q, \dot{q}) \phi \triangleq G(q)+F_{s} \operatorname{sgn}(\dot{q})
$$

where $\phi \in \mathbb{R}^{p}$ contains unknown constant parameters, and the regression matrix $Y(q, \dot{q}) \in \mathbb{R}^{n \times p}$ contains measurable functions of the link position and link velocity. Lower and upper bounds denoted by $\phi, \bar{\phi} \in \mathbb{R}^{p}$, respectively, are assumed to be known for each parameter in $\phi$ as follows:

$$
\underline{\phi_{i}} \leq \phi_{i} \leq \overline{\phi_{i}} \quad \forall i=1,2, \ldots p
$$

where $\underline{\phi_{i}} \overline{\phi_{i}} \in \mathbb{R}$ denote the $i$-th component of $\underline{\phi}$ and $\bar{\phi}$, respectively, and $\phi_{i} \bar{\in} \mathbb{R}$ denotes the $i$ th component of $\phi$.

Property 4: The time derivative of the inertia matrix, the centripetalCoriolis matrix, the gravity vector, and the static friction matrix can be upper bounded in the following manner:

$$
\begin{aligned}
& \|\dot{M}(q)\|_{i \infty} \leq \zeta_{m}\|\dot{q}\| \quad\left\|V_{m}(q, \dot{q})\right\|_{i \infty} \leq \zeta_{c}\|\dot{q}\| \\
& \|M(q)\|_{i \infty} \leq \zeta_{m 2} \quad\|G(q)\| \leq \zeta_{g} \quad\left\|F_{s}\right\| \leq \zeta_{f}
\end{aligned}
$$

where $\zeta_{g}, \zeta_{f}, \zeta_{c}$, and $\zeta_{m}, \zeta_{m 2} \in \mathbb{R}$ are known positive constants, and $\|\cdot\|_{i \infty}$ denotes the induced infinity norm of a matrix.

Property 5: Since the controller in this note is developed for revolute robots, the terms $M(q), V_{m}(q, \dot{q}), G(q)$, and $J(q)$ are bounded for all possible $q(t)$. That is, these terms only depend on $q(t)$ as arguments of bounded trigonometric functions, and

$$
\|J(q)\|_{i \infty}<\delta_{1}
$$

where $\delta_{1} \in \mathbb{R}$ is a known positive constant. The subsequent development is also based on the assumption that all kinematic singularities associated with $J(q)$ are assumed to always be avoided.

Property 6: The product of the manipulator Jacobian with any measurable vector $\xi(t) \in \mathbb{R}^{m}$ can be linearly parameterized as

$$
Y_{J}(q, \xi) \phi_{J} \triangleq J^{T}(q) \xi
$$

where $Y_{J}(q, \xi) \in \mathbb{R}^{n \times p_{2}}$ contains measurable functions of the link position and $\xi$, and $\phi_{J} \in \mathbb{R}^{p_{2}}$ contains the unknown constant parameters contained in the Jacobian matrix. Lower and upper bounds denoted by $\phi_{J}, \overline{\phi_{J}} \in \mathbb{R}^{p_{2}}$, respectively, are assumed to be known for each parameter in $\phi_{J}$ as

$$
\underline{\phi_{J k}} \leq \phi_{J k} \leq \overline{\phi_{J k}} \quad \forall k=1,2, \ldots p_{2}
$$

where $\phi_{J k}, \overline{\phi_{J k}} \in \mathbb{R}$ denote the $k$-th component of $\underline{\phi_{J}}$ and $\overline{\phi_{J}}$, respectively, and $\phi_{J k} \in \mathbb{R}$ denotes the $k$ th component of $\overline{\phi_{J}}$.

\section{CONTROL DEVELOPMENT}

\section{A. Control Objective}

Many robotic tasks are naturally defined in terms of the task-space. The objective for these tasks is to regulate the end-effector of a robot manipulator to a desired task-space setpoint. To quantify this objective, a task-space setpoint error denoted by $e(t) \in \mathbb{R}^{m}$ is defined as

$$
e \triangleq x-x_{d}
$$

where $x(t)$ was introduced in (3), and $x_{d} \in \mathbb{R}^{m}$ denotes the known, constant desired setpoint.

Unlike typical task-space regulation results, a contribution of this note is that the constant parameters in the manipulator Jacobian are not assumed to be known (i.e., the task-space to joint-space relationship may be corrupt). To develop a controller that allows for task-space regulation in the presence of uncertain kinematic and dynamic parameters, the subsequent development is based on the assumption that $x(t)$, $q(t)$, and $\dot{q}(t)$ are measurable. Specifically, $q(t)$ and $\dot{q}(t)$ can be obtained from encoder/tachometer sensors, and $x(t)$ could be obtained from a camera system [3].

\section{B. Control Development}

Based on the control objective and the subsequent stability analysis, the following adaptive controller is developed:

$$
\tau=Y(q, \dot{q}) \hat{\phi}-k_{p} \hat{J}^{T}(q) \operatorname{Tanh}(e)-k_{v} \operatorname{Tanh}(\dot{q})
$$

where $k_{v}, k_{p} \in \mathbb{R}$ denote constant control gains, $\hat{\phi}(t) \in \mathbb{R}^{p}$ denotes a subsequently designed parameter estimate, $\hat{J}(q) \in \mathbb{R}^{m \times n}$ represents a subsequently designed Jacobian estimate, and the vector functions $\operatorname{Tanh}(e) \in \mathbb{R}^{m}$ and $\operatorname{Tanh}(\dot{q}) \in \mathbb{R}^{n}$ are defined as

$$
\begin{aligned}
& \operatorname{Tanh}(e) \triangleq\left[\tanh \left(e_{1}\right), \tanh \left(e_{2}\right), \ldots, \tanh \left(e_{m}\right)\right]^{T} \\
& \operatorname{Tanh}(\dot{q}) \triangleq\left[\tanh \left(\dot{q}_{1}\right), \tanh \left(\dot{q}_{2}\right), \ldots, \tanh \left(\dot{q}_{n}\right)\right]^{T} .
\end{aligned}
$$


To facilitate the design of the Jacobian estimate, the linear parameterization $Y_{J}(q, e) \phi_{J}$ introduced in Property 6 is specifically defined as

$$
Y_{J}(q, e) \phi_{J} \triangleq J^{T}(q) \operatorname{Tanh}(e)
$$

where $Y_{J}(q, e) \in \mathbb{R}^{n \times p_{2}}$ contains measurable functions of the link position and task-space setpoint error, and $\phi_{J} \in \mathbb{R}^{p_{2}}$ contains the unknown constant parameters contained in the Jacobian matrix. An estimate for (12) is developed as

$$
Y_{J}(q, e) \hat{\phi}_{J} \triangleq \hat{J}^{T}(q) \operatorname{Tanh}(e)
$$

where $\hat{\phi}_{J}(t) \in \mathbb{R}^{p_{2}}$ denotes a parameter estimate. Based on the subsequent stability analysis, the parameter estimates $\hat{\phi}(t)$ and $\hat{\phi}_{J}(t)$ are generated from the following adaptation laws:

$$
\dot{\hat{\phi}}_{i}=\operatorname{proj}\left\{\Omega_{o i}\right\} \quad \dot{\hat{\phi}}_{J k}(t)=\operatorname{proj}\left\{\Omega_{1 k}\right\}
$$

where $\Omega_{o i}(q, \dot{q}, e)$ and $\Omega_{1 k}(q, \dot{q}, e)$ denote the $i$ th and $k$ th component of $\Omega_{o}(q, \dot{q}, e)$ and $\Omega_{1}(q, \dot{q}, e)$, respectively, $\forall i=1,2, \ldots p$ and $\forall k=1,2, \ldots p_{2}$, where the auxiliary terms $\Omega_{o}(q, \dot{q}, e) \in \mathbb{R}^{p}$ and $\Omega_{1}(q, \dot{q}, e) \in \mathbb{R}^{p_{2}}$ are defined as

$$
\begin{aligned}
& \Omega_{o}(q, \dot{q}, e) \triangleq-\Gamma_{0} Y^{T}(q, \dot{q})\left(\dot{q}+\varepsilon Y_{J}(q, e) \hat{\phi}_{J}\right) \\
& \Omega_{1}(q, \dot{q}, e) \triangleq k_{p} \Gamma_{1} Y_{J}^{T}(q, e) \dot{q} .
\end{aligned}
$$

For the adaptation laws given in (14) and (15), $\Gamma_{0} \in \mathbb{R}^{p \times p}$ and $\Gamma_{1} \in$ $\mathbb{R}^{p_{2} \times p_{2}}$ denote constant, diagonal positive definite adaptation gain matrices, $\varepsilon \in \mathbb{R}$ is a positive, constant adaptation weighting gain, and the function $\operatorname{proj}\{\cdot\}$ is defined as follows:

$$
\begin{array}{r}
\operatorname{proj}\left\{\Omega_{o i}\right\} \triangleq\left\{\begin{array}{lll}
\Omega_{o i}, & \text { if } \hat{\phi}_{i}>\underline{\phi_{i}} \\
\Omega_{o i}, & \text { if } \hat{\phi}_{i}=\underline{\phi_{i}} & \text { and } \quad \Omega_{o i} \geq 0 \\
0 & \text { if } \hat{\phi}_{i}=\overline{\phi_{i}} & \text { and } \quad \Omega_{o i}<0 \\
0, & \text { if } \hat{\phi}_{i}=\overline{\overline{\phi_{i}}} \text { and } \quad \Omega_{o i}>0 \\
\Omega_{o i} & \text { if } \hat{\phi}_{i}=\overline{\phi_{i}} & \text { and } \quad \Omega_{o i} \leq 0 \\
\Omega_{o i}, & \text { if } \hat{\phi}_{i}<\overline{\phi_{i}}
\end{array}\right. \\
\underline{\phi_{i} \leq \hat{\phi}_{i}(0) \leq \overline{\phi_{i}}}
\end{array}
$$

where $\hat{\phi}_{i}(t)$ denotes the $i$ th component of $\hat{\phi}(t)$. The $\operatorname{proj}\left\{\Omega_{1 k}(q, \dot{q}, e)\right\}$ is defined in the same manner as in (16) with regard to $\hat{\phi}_{J k}(t)$. Based on the aforementioned assumption that all kinematic singularities associated with $J(q)$ are always be avoided, the development for $\hat{J}(q)$ can be used to determine that $\hat{J}(q)$ is also nonsingular; hence, [20, Th. 5.9] can be used to conclude that

$$
0<\lambda_{\min }\left\{\hat{J}(q) \hat{J}^{T}(q)\right\} .
$$

The closed-loop error system can be determined after substituting (11) into (2) as follows:

$$
M(q) \ddot{q}+V_{m}(q, \dot{q}) \dot{q}=-Y(q, \dot{q}) \tilde{\phi}-k_{p} Y_{J}(q, e) \hat{\phi}_{J}-k_{v} \operatorname{Tanh}(\dot{q})
$$

where (6) and (13) have been utilized. In (17), the parameter estimation error signal $\tilde{\phi}(t) \in \mathbb{R}^{p}$ is defined as

$$
\tilde{\phi} \triangleq \phi-\hat{\phi}
$$

and a parameter estimation error signal $\tilde{\phi}_{J}(t) \in \mathbb{R}^{p_{2}}$ is also defined for the mismatch between the actual and estimated Jacobian parameters as follows:

$$
\tilde{\phi}_{J} \triangleq \phi_{J}-\hat{\phi}_{J}
$$

Based on the control development introduced in (11)-(16), the following properties can be developed to facilitate the stability analysis.
Property 7: The projection algorithm in (16) ensures that the following inequalities are satisfied (for further details, see [2] and [18]):

$$
\underline{\phi_{i}} \leq \hat{\phi}_{i}(t) \leq \overline{\phi_{i}} \quad \underline{\phi_{J k}} \leq \hat{\phi}_{J k}(t) \leq \overline{\phi_{J k}} .
$$

Based on (14)-(16), the following inequality can also be shown to hold:

$$
\left\|\dot{\hat{\phi}}_{J}\right\| \leq\left\|\Omega_{1}(q, \dot{q}, e)\right\| \leq k_{p} \lambda_{\max }\left\{\Gamma_{1}\right\}\left\|Y_{J}(q, e)\right\|_{i \infty}\|\dot{q}\|
$$

where $\lambda_{\max }\{\cdot\}$ denotes the maximum eigenvalue of a matrix.

Property 8: From (9), (12), (13), and (20), the estimated Jacobian matrix can be upper bounded as

$$
\|\hat{J}(q)\|_{i \infty}<\delta_{2}
$$

where $\delta_{2} \in \mathbb{R}$ denotes a known positive constant (i.e., $\delta_{2}>0$ ).

Property 9: The time derivative of (12) can be upper bounded as

$$
\left\|\frac{d}{d t}\left(Y_{J}(q, e) \hat{\phi}_{J}(t)\right)\right\| \leq \zeta_{J}\|\dot{q}\|
$$

where $\zeta_{J} \in \mathbb{R}$ denotes a known positive constant.

Property 10: The following inequalities can be shown to hold for all $e(t) \in \mathbb{R}^{m}$ and $\dot{q}(t) \in \mathbb{R}^{n}[10]$

$$
2 \sum_{i=1}^{m} \ln \left(\cosh \left(e_{i}\right)\right) \geq\|\operatorname{Tanh}(e)\|^{2} \geq \tanh ^{2}(\|e\|)
$$

$$
\|\dot{q}\|+1 \geq \frac{\|\dot{q}\|}{\tanh (\|\dot{q}\|)}
$$

$\|\operatorname{Tanh}(e)\|\|\operatorname{Tanh}(\dot{q})\| \leq\|\operatorname{Tanh}(e)\|^{2}+\|\operatorname{Tanh}(\dot{q})\|^{2}$

$$
\|\operatorname{Tanh}(e)\|\|\dot{q}\| \leq\|\operatorname{Tanh}(e)\|^{2}+\|\dot{q}\|^{2}
$$

$$
\|\dot{q}\| \geq\|\operatorname{Tanh}(\dot{q})\|
$$

where $\ln (\cdot)$ denotes the natural logarithm.

Property 11: The control effort can be upper bounded in terms of $a$ priori known terms as

$$
\|\tau\| \leq\|Y\|_{i \infty}\|\bar{\phi}\|+k_{p}\left\|Y_{J}\right\|_{i \infty}\left\|\bar{\phi}_{J}\right\|+k_{v} \sqrt{n}
$$

where the control gains $k_{v}$ and $k_{p}$ can be made arbitrarily small provided some relative magnitudes are maintained as subsequently described.

Property 12: Based on (13) and (14)-(16), the following inequality can be developed [3]:

$$
\begin{aligned}
\lambda_{\min }\left\{\hat{J}(q) \hat{J}^{T}\right. & (q)\}\|\operatorname{Tanh}(e)\|^{2} \\
& \leq \operatorname{Tanh}^{T}(e) \hat{J}(q) \hat{J}^{T}(q) \operatorname{Tanh}(e) \quad \forall e(t) \in \mathbb{R}^{m}
\end{aligned}
$$

where $\lambda_{\min }\{\cdot\}$ denotes the minimum eigenvalue of the argument.

\section{STABILITy ANALYSis}

Theorem 1: Given the robotic system defined by (2) and (3), the control torque input given in (11), along with the adaptation law given in (14)-(16) ensures semi-global asymptotic regulation of the task-space error in the sense that

$$
\|e(t)\| \rightarrow 0, \quad \text { as } \quad t \rightarrow \infty .
$$

The result in (31) is valid, provided the control gains $k_{p}$ and $k_{v}$ given in (11)-(15), and the adaptation weighting gain $\varepsilon$ defined in (15) are chosen to satisfy the following sufficient conditions:

$$
k_{p}>k_{v} \frac{\delta_{2}}{\lambda_{\min }\left\{\hat{J}(q) \hat{J}^{T}(q)\right\}}>0
$$



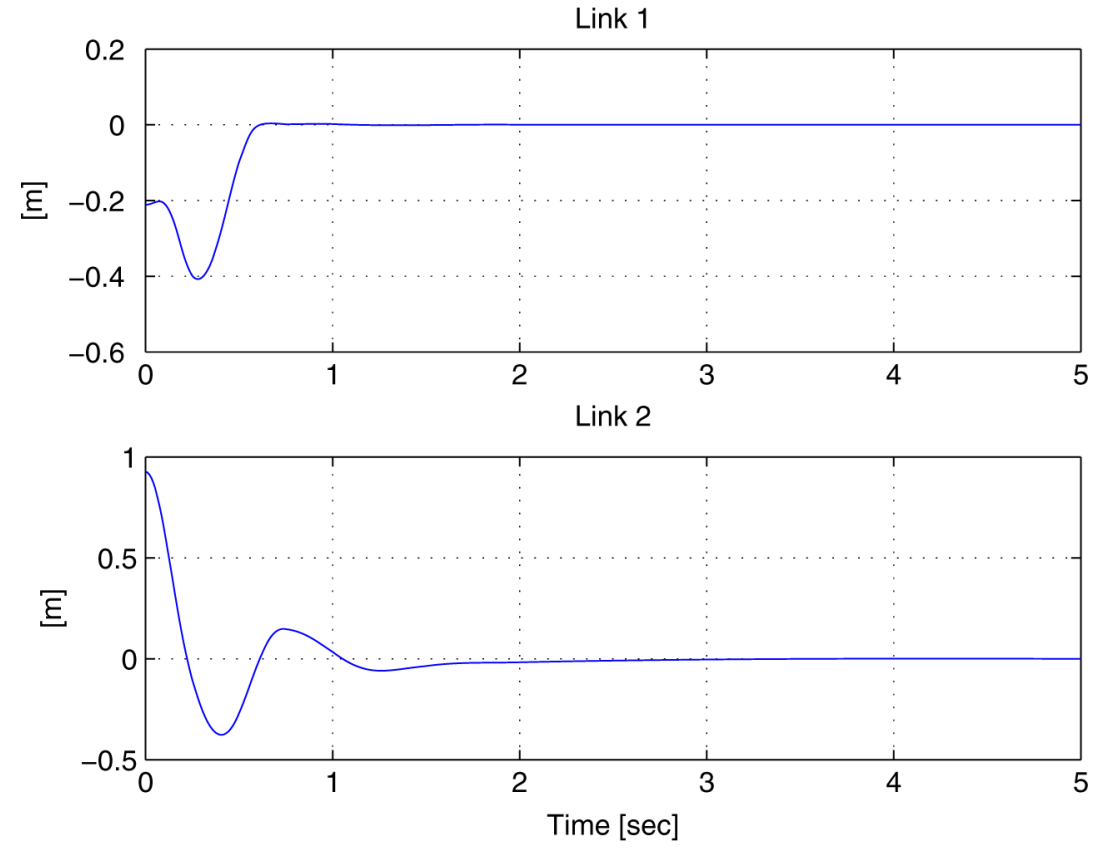

Fig. 1. Task-space position error.

and

$$
\varepsilon<\min \left\{\frac{m_{1}}{2 \delta_{2} m_{2}}, \frac{k_{p}}{2 \delta_{2} m_{2}}, \frac{1}{2 \delta_{2}}\right\}
$$

$$
\frac{k_{v}\left(1-2 \varepsilon \delta_{2}\right)}{2 \varepsilon \zeta_{\chi}} \geq\left[\sqrt{\frac{\lambda_{2}(0)}{\frac{1}{2} m_{1}-\varepsilon \delta_{2} m_{2}}}+1\right]^{2}
$$

where $m_{1}$ and $m_{2}$ are defined in (4), $\delta_{2}$ was defined in (22), $\zeta_{\chi} \in \mathbb{R}$ denotes a positive bounding constant, and $\lambda_{2}(t) \in \mathbb{R}$ denotes a subsequently defined state-dependent positive bounding function.

Remark 1: The adaptive weighting gain $\varepsilon$ can be selected arbitrarily small to satisfy the conditions given in (32)-(34). Hence, the magnitude of $k_{v}$ (and thus $k_{p}$ ) can be made arbitrarily small, provided the combination of the gains is selected according to the initial conditions of the states of the system due to the term $\lambda_{2}(0)$ in (34). Since the selection of the control gains depends on the initial conditions of the states, the result in (31) is semiglobal.

Proof: Let $V(t) \in \mathbb{R}$ denote the following nonnegative function (i.e., a Lyapunov function candidate):

$$
\begin{aligned}
V(t) \triangleq & \frac{1}{2} \dot{q}^{T} M(q) \dot{q}+\varepsilon \operatorname{Tanh}^{T}(e) \hat{J}(q) M(q) \dot{q} \\
& +\sum_{i=1}^{m} k_{p} \ln \left(\cosh \left(e_{i}\right)\right)+\frac{1}{2} \tilde{\phi}^{T} \Gamma_{0}^{-1} \tilde{\phi}+\frac{1}{2} \tilde{\phi}_{J}^{T} \Gamma_{1}^{-1} \tilde{\phi}_{J}
\end{aligned}
$$

The Raleigh-Ritz Theorem [11] can be used along with (4), (24), and (27) to bound (35) by the following inequalities:

$$
\lambda_{1}(t) \leq V(t) \leq \lambda_{2}(t)
$$

In (36), the positive functions $\lambda_{1}(t), \lambda_{2}(t) \in \mathbb{R}$ are defined as

$$
\begin{aligned}
\lambda_{1}(t) \triangleq & \left(\frac{1}{2} m_{1}-\varepsilon \delta_{2} m_{2}\right)\|\dot{q}\|^{2} \\
& +\sum_{i=1}^{m}\left(k_{p}-2 \varepsilon \delta_{2} m_{2}\right) \ln \left(\cosh \left(e_{i}\right)\right) \\
& +\frac{1}{2} \lambda_{\min }\left\{\Gamma_{0}^{-1}\right\}\|\tilde{\phi}\|^{2}+\frac{1}{2} \lambda_{\min }\left\{\Gamma_{1}^{-1}\right\}\left\|\tilde{\phi}_{J}\right\|^{2}
\end{aligned}
$$

$$
\begin{aligned}
\lambda_{2}(t) \triangleq & \left(\frac{1}{2}+\varepsilon \delta_{2}\right) m_{2}\|\dot{q}\|^{2} \\
& +\sum_{i=1}^{m}\left(k_{p}+2 \varepsilon \delta_{2} m_{2}\right) \ln \left(\cosh \left(e_{i}\right)\right) \\
& +\frac{1}{2} \lambda_{\max }\left\{\Gamma_{0}^{-1}\right\}\|\tilde{\phi}\|^{2}+\frac{1}{2} \lambda_{\max }\left\{\Gamma_{1}^{-1}\right\}\left\|\tilde{\phi}_{J}\right\|^{2}
\end{aligned}
$$

Based on (37), it is straightforward that if $\varepsilon$ is selected according to (33), then $\lambda_{1}(t) \geq 0$; hence, from (36) $V(t) \geq 0$.

After taking the time derivative of (35), the following simplified expression can be obtained:

$$
\begin{aligned}
\dot{V}(t)= & -\dot{q}^{T} Y \tilde{\phi}-\varepsilon \operatorname{Tanh}^{T}(e) \hat{J}(q) Y \tilde{\phi} \\
& +k_{p} \dot{q}^{T} Y_{J} \tilde{\phi}_{J}-k_{v} \dot{q}^{T} \operatorname{Tanh}(\dot{q})+\varepsilon \chi \\
& -\varepsilon k_{p} \hat{\phi}_{J}^{T} Y_{J}^{T} Y_{J} \hat{\phi}_{J}-\varepsilon k_{v} \hat{\phi}_{J}^{T} Y_{J}^{T} \operatorname{Tanh}(\dot{q}) \\
& -\tilde{\phi}^{T} \Gamma_{0}^{-1} \dot{\hat{\phi}}-\tilde{\phi}_{J}^{T} \Gamma_{1}^{-1} \dot{\hat{\phi}}_{J}
\end{aligned}
$$

where (5), (12), (13), (17), and (18) were utilized, and the auxiliary term $\chi(t) \in \mathbb{R}$ is defined as

$$
\chi \triangleq \frac{d}{d t}\left(Y_{J} \hat{\phi}_{J}\right) M(q) \dot{q}+\hat{\phi}_{J}^{T} Y_{J}^{T}\left(\dot{M}(q)-V_{m}(q, \dot{q})\right) \dot{q} .
$$

Based on the form of (40), Properties 4, 5, and 8-10 can be utilized to show that

$$
\|\chi\| \leq \zeta_{\chi}\|\dot{q}\|^{2}
$$

where the positive constant $\zeta_{\chi}$ was introduced in (34). After utilizing (13), (15), (22), (26)-(30), and (41), the following expression can be obtained:

$$
\begin{aligned}
\dot{V}(t) \leq & -\varepsilon k_{p} \lambda_{\min }\left\{\hat{J} \hat{J}^{T}\right\}\|\operatorname{Tanh}(e)\|^{2}-k_{v}\|\operatorname{Tanh}(\dot{q})\|^{2} \\
& +\varepsilon k_{v} \delta_{2}\left(\|\operatorname{Tanh}(e)\|^{2}+\|\operatorname{Tanh}(\dot{q})\|^{2}\right)+\varepsilon \zeta_{\chi}\|\dot{q}\|^{2} \\
& +\tilde{\phi}^{T} \Gamma_{0}^{-1}\left(\Omega_{o}-\dot{\hat{\phi}}\right)+\tilde{\phi}_{J}^{T} \Gamma_{1}^{-1}\left(\Omega_{1}-\dot{\hat{\phi}}_{J}\right)
\end{aligned}
$$


Link 1
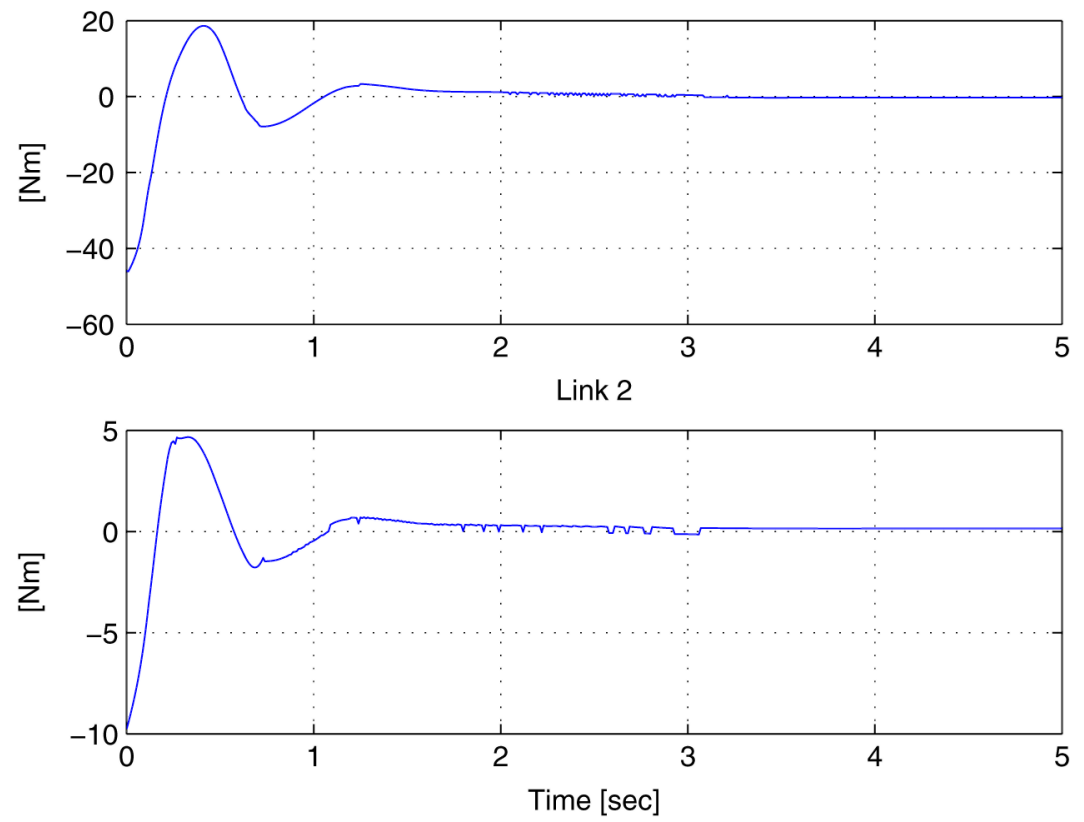

Fig. 2. Control torque inputs.

By utilizing (14)-(16), the following advantageous expression can be developed for the upper bound of (42):

$$
\begin{aligned}
\dot{V}(t) \leq & -\frac{k_{v}}{2}\|\operatorname{Tanh}(\dot{q})\|^{2} \\
& -\varepsilon\left(k_{p} \lambda_{\min }\left\{\hat{J} \hat{J}^{T}\right\}-k_{v} \delta_{2}\right)\|\operatorname{Tanh}(e)\|^{2} \\
& -\left(\frac{k_{v}}{2}-\varepsilon k_{v} \delta_{2}\right)\|\operatorname{Tanh}(\dot{q})\|^{2}+\varepsilon \zeta_{\chi}\|\dot{q}\|^{2} .
\end{aligned}
$$

Provided that the condition given in (32) and the following inequality are both satisfied:

$$
-\left(\frac{k_{v}}{2}-\varepsilon k_{v} \delta_{2}\right)\|\operatorname{Tanh}(\dot{q})\|^{2}+\varepsilon \zeta_{\chi}\|\dot{q}\|^{2} \leq 0
$$

the expression in (43) can be used to prove that $\dot{V}(t) \leq 0$. To facilitate further analysis, (25), (36), and (37) are used to obtain a sufficient condition for (44) as

$$
\frac{\left(k_{v}-2 \varepsilon k_{v} \delta_{2}\right)}{2 \varepsilon \zeta_{\chi}} \geq\left(\sqrt{\frac{V(t)}{\frac{1}{2} m_{1}-\varepsilon \delta_{2} m_{2}}}+1\right)^{2} .
$$

If the conditions in (32), (33), and (45) are satisfied, the inequality in (43) can be used to obtain the following inequality:

$$
\dot{V}(t) \leq-\beta\|\psi\|^{2}
$$

where $\beta \in \mathbb{R}$ is a positive constant, and $\psi(t) \in \mathbb{R}^{m+n}$ is

$$
\psi \triangleq\left[\operatorname{Tanh}^{T}(e) \operatorname{Tanh}^{T}(\dot{q})\right]^{T} .
$$

From (46), it is clear that $\dot{V}(t) \leq 0$; therefore

$$
V(z(t), t) \leq V(z(0), 0) \leq \lambda_{2}(z(0), 0) \quad \forall t \geq 0
$$

where $\lambda_{2}(t)$ was defined in (38), and $z(t) \in \mathbb{R}^{4}$ is given by

$$
z \triangleq\left[\begin{array}{llll}
\|\dot{q}\|^{2} & \sum_{i=1}^{m} \ln \left(\cosh \left(e_{i}\right)\right) \quad\|\tilde{\phi}\|^{2} & \left\|\tilde{\phi}_{J}\right\|^{2}
\end{array}\right]^{T} .
$$

Based on (48), the final sufficient condition for (45) can be expressed by the inequality in (34).

From (48), it is clear that $V(t) \in \mathcal{L}_{\infty}$; hence, $\dot{q}(t), e(t), \tilde{\phi}(t)$, $\tilde{\phi}_{J}(t), \psi(t) \in \mathcal{L}_{\infty}$. Since $e(t) \in \mathcal{L}_{\infty}$, and the desired setpoint is constant, it is clear that $x(t) \in \mathcal{L}_{\infty}$. Since the development is directed at revolute robots, $q(t)$ only appears in $h(q)$ in (3) as an argument of bounded trigonometric functions; hence, it is typically unclear how the boundedness of $q(t)$ can be proven. However, the boundedness of $q(t)$ is typically not a concern since $q(t)$ only appears as an argument of bounded trigonometric functions in the controller. From (18), (14)-(16), (7), and the preceding arguments, it is clear that $\hat{\phi}(t)$, $\hat{\phi}(t), \hat{\phi}_{J}(t), \hat{\phi}_{J}(t), \tau(t) \in \mathcal{L}_{\infty}$. Moreover, (3) and the facts that $\dot{q}(t)$, $J(q) \in \mathcal{L}_{\infty}$ can be used to prove that $\dot{x}(t), \dot{e}(t) \in \mathcal{L}_{\infty}$; hence, $e(t)$ is uniformly continuous. From (46), (47), and the properties of the hyperbolic tangent, it is clear that $\dot{q}(t), e(t) \in \mathcal{L}_{2}$ [11]. Since $e(t)$, $\dot{e}(t) \in \mathcal{L}_{\infty}$ and $e(t) \in \mathcal{L}_{2}$, Barbalat's Lemma [29] can be invoked to conclude the result in (31).

\section{Simulation}

The controller developed in (11) and the adaptation law given in (14)-(16) were simulated for a two-link robot planar manipulator. The manipulator Jacobian is given by

$$
J=\left[\begin{array}{cc}
-L_{1} \sin \left(q_{1}\right)-L_{2} \sin \left(q_{1}+q_{2}\right) & -L_{2} \sin \left(q_{1}+q_{2}\right) \\
L_{1} \cos \left(q_{1}\right)+L_{2} \cos \left(q_{1}+q_{2}\right) & L_{2} \cos \left(q_{1}+q_{2}\right)
\end{array}\right]
$$

where $L_{1}$ and $L_{2}$ denote unknown link lengths. The dynamics of the planar manipulator are

$$
\tau=M(q) \ddot{q}+V_{m}(q, \dot{q}) \dot{q}+F_{s} \operatorname{sgn}(\dot{q}) .
$$

The vectors of uncertain constant kinematic and dynamic parameters, $\phi_{J} \in \mathbb{R}^{2}$ and $\phi \in \mathbb{R}^{2}$, respectively, was found to be

$$
\phi_{J}=\left[\begin{array}{ll}
L_{1} & L_{2}
\end{array}\right] \quad \phi=\left[\begin{array}{ll}
F_{s 1} & F_{s 2}
\end{array}\right]
$$

where $F_{s 1}, F_{s 2}$ denote diagonal elements of $F_{s}$, and the initial parameter estimates were selected to be $20 \%$ of the actual values. The desired 
task-space setpoints (in $[\mathrm{m}]$ ) and corresponding desired joint-space setpoints (in $[\mathrm{deg}]$ ) were selected as

$$
x_{d}=\left[\begin{array}{ll}
2.6 & -0.08
\end{array}\right] \quad q_{d}=\left[\begin{array}{ll}
0 & -5.7
\end{array}\right]
$$

where

$$
x(0)=\left[\begin{array}{ll}
2.38 & 0.84
\end{array}\right] \quad q(0)=\left[\begin{array}{ll}
28.65 & -28.65
\end{array}\right] .
$$

By adjusting the control gains as

$$
\begin{aligned}
k_{p} & =\operatorname{diag}\{120,80\} \quad k_{v}=\operatorname{diag}\{1,1\} \quad \varepsilon=0.005 \\
\Gamma_{0} & =\operatorname{diag}\{0.35,0.025\} \quad \Gamma_{1 J}=\operatorname{diag}\{0.015,0.0015\} .
\end{aligned}
$$

the results given in Figs. 1 and 2 can be obtained. Specifically, the task-space error is depicted in Fig. 1. Fig. 2 shows the control input torques. Fig. 1 illustrates that the developed adaptive controller yields asymptotic regulation of the robot to a desired constant task-space setpoint despite uncertainties throughout the manipulator kinematics and dynamics.

\section{CONCLUSION}

An amplitude limited controller was developed for robot manipulators despite uncertainty in the dynamic and kinematic models. The adaptive controller yields semi-global asymptotic regulation of the task-space error. The advantages of the proposed controller include the ability to actively compensate for unknown parametric effects in the dynamic and kinematic model and the ability to a priori calculate the maximum required torque.

\section{REFERENCES}

[1] S. Arimoto, "Robotics research toward explication of everyday physics," Int. J. Rob. Res., vol. 18, no. 11, pp. 1056-1063, 1999.

[2] M. Bridges, D. M. Dawson, and C. Abdallah, "Control of rigid-link flexible-joint robots: A survey of backstepping approaches," J. Rob. Syst., vol. 12, pp. 199-216, 1995.

[3] C. Cheah, M. Hirano, S. Kawamura, and S. Arimoto, "Approximate Jacobian control for robots with uncertain kinematics and dynamics," IEEE Trans. Robot. Automat., vol. 19, no. 4, pp. 692-702, Jul. 2003.

[4] C. Cheah, S. Kawamura, and S. Arimoto, "Feedback control for robotic manipulators with uncertain kinematics and dynamics," in Proc. IEEE Int. Conf. Robot. Automat., 1998, pp. 3607-3612.

[5] —_ "Feedback control for robotic manipulators with an uncertain Jacobian matrix," Int. J. Rob. Syst., vol. 12, no. 2, pp. 119-134, 1999.

[6] C. Cheah, S. Kawamura, S. Arimoto, and K. Lee, "PID control for robotic manipulator with uncertain Jacobian matrix," in Proc. IEEE Int. Conf. Robot. Automat., 1999, pp. 494-499.

[7] J. Chen, D. M. Dawson, W. E. Dixon, and V. Chitrakaran, "An optimization-based approach for fusing image-based trajectory generation with position-based visual servo control," in Proc. IEEE Conf. Decision Conttrol, 2004, pp. 4034-4039.

[8] R. Colbaugh, E. Barany, and K. Glass, "Global regulation of uncertain manipulators using bounded controls," in Proc. IEEE Int. Conf. Robot. Automat., 1997, pp. 1148-1155.

[9] — "Global stabilization of uncertain manipulators using bounded controls," in Proc. IEEE Amer. Control Conf., 1997, pp. 86-91.

[10] W. E. Dixon, M. S. de Queiroz, D. M. Dawson, and F. Zhang, "Tracking control of robot manipulators with bounded torque inputs," Robotica, vol. 17, pp. 121-129, 1999.

[11] W. E. Dixon, A. Behal, D. M. Dawson, and S. Nagarkatti, Nonlinear Control of Engineering Systems: A Lyapunov-Based Approach. Boston, MA: Birkhäuser, 2003.

[12] W. E. Dixon, "Adaptive regulation of amplitude limited robot manipulators with uncertain kinematics and dynamics," in Proc. IEEE Amer. Control Conf., 2004, pp. 3839-3844.
[13] R. Kelly and V. Santibáñez, "A class of global regulators with bounded control actions for robot manipulators," in Proc. IEEE Conf. Decision Control, 1996, pp. 3382-3387.

[14] R. Kelly, J. Moreno, and R. Campa, "Visual servoing of planar robots via velocity fields," in Proc. IEEE Conf. Decision Control, 2004, pp. 4028-4033.

[15] F. Lewis, C. Abdallah, and D. Dawson, Control of Robot Manipulators. New York: MacMillan, 1993.

[16] A. Loria, R. Kelly, and R. Ortega, "On global output feedback regulation of Euler-lagrange systems with bounded inputs," in Proc. IFAC World Congr., 1996, pp. 161-166.

[17] A. Loria and H. Nijmeijer, "Bounded output feedback tracking control of fully-actuated Euler-lagrange systems," Syst. Control Lett., vol. 33, no. 3, pp. 151-161, 1998.

[18] R. Lozano and B. Brogliato, "Adaptive control of robot manipulators with flexible joints," IEEE Trans. Autom. Conrol, vol. 37, no. 2, pp. 174-181, Feb. 1992.

[19] R. Ortega, A. Loria, and R. Kelly, "A semiglobally stable output feedback $\mathrm{PI}^{2} \mathrm{D}$ regulator for robot manipulators," IEEE Trans. Autom. Control, vol. 40, no. 8, pp. 1432-1436, Aug. 1995.

[20] S. Perlis, Theory of Matrices. New York: Dover, 1991.

[21] J. A. Piepmeier, G. V. McMurray, and H. Lipkin, "A dynamic quasiNewton method for uncalibrated visual servoing," in Proc. IEEE Int. Conf. Robot. Autom., 1999, pp. 1595-1600.

[22] _ _ "A dynamic Jacobian estimation method for uncalibrated visual servoing," in Proc. IEEE Int. Conf. Adv. Int. Mech., 1999, pp. 944-949.

[23] J. A. Piepmeier, B. A. Gumpert, and H. Lipkin, "Uncalibrated eye-inhand visual servoing," in Proc. IEEE Int. Conf. Robot. Automat., 2002, pp. 568-573.

[24] J. A. Piepmeier, "Experimental results for uncalibrated eye-in-hand visual servoing," in South. Symp. Syst. Theory, 2003, pp. 335-339.

[25] J. A. Piepmeier, G. V. McMurray, and H. Lipkin, "Uncalibrated dynamic visual servoing," IEEE Trans. Robot. Automat., vol. 20, no. 1, pp. 143-147, Jan. 2004.

[26] V. Santibáñez and R. Kelly, "Global regulation for robot manipulators under SP-SD feedback," in Proc. IEEE Int. Conf. Robot. Automat., 1996, pp. 927-932.

[27] S. Sastry and M. Bodson, Adaptive Control: Stability, Convergence, and Robustness. Englewood Cliffs, NJ: Prentice-Hall, 1989.

[28] M. Shahamiri and M. Jagersand, "Uncalibrated visual servoing using a biased Newton method for on-line singularity detection and avoidance," in Proc. IEEE/RSJ Int. Conf. Int. Rob. Syst., 2005, pp. 3953-3958.

[29] J. J. E. Slotine and W. Li, Applied Nonlinear Control. Englewood Cliffs, NJ: Prentice-Hall, 1991

[30] E. Zergeroglu, W. E. Dixon, A. Behal, and D. M. Dawson, "Adaptive set-point control of robotic manipulators with amplitude-limited control inputs," Robotica, vol. 18, no. 2, pp. 171-181, 2000.

\section{Estimation of Sinusoidal Frequencies and Amplitudes Using Adaptive Identifier and Observer}

\author{
M. Hou
}

\begin{abstract}
A simple method is proposed for estimation of amplitudes of multiple sinusoids. The estimation is based on the existing adaptive identifier which offers the globally convergent estimate of sinusoidal frequencies. To deal with possible singularities of the amplitude estimation, adaptive observers are also proposed for estimation of sinusoidal amplitudes. Simulations illustrate the results.
\end{abstract}

Index Terms-Adaptive identifier, adaptive observer, frequency and amplitude estimation, signal processing.

Manuscript received October 18, 2005; revised May 18, 2005 and September 4, 2006. Recommended by Associate Editor W. X. Zheng.

The author is with the Department of Engineering, the University of Hull, Hull HU6 7RX, U.K. (e-mail: m.hou@ hull.ac.uk).

Digital Object Identifier 10.1109/TAC.2006.890389 